\title{
The efficacy and safety of the Chinese herbal medicine Di-Tan decoction for treating Alzheimer's disease: protocol for a randomized controlled trial
}

\author{
Ka-Kit Chua ${ }^{1}$, Adrian Wong ${ }^{2}$, Pauline Wing-Lam Kwan², Ju-Xian Song ${ }^{1}$, Lei-Lei Chen ${ }^{1}$, Andrew Lung-Tat Chan ${ }^{3}$, \\ Jia-Hong Lu', Vincent Mok ${ }^{2^{*}}$ and Min Li ${ }^{1^{*}}$
}

\begin{abstract}
Background: Alzheimer's disease (AD) is the most common type of dementia in the elderly. It is estimated that the global prevalence of dementia will rise from 24.3 million in 2005 to 81.1 million in 2040. AD has a devastating impact on sufferers, caregivers, their communities and the healthcare system in general. "Di-tan decoction" (DTD) is a traditional Chinese medicine (TCM) formula frequently used to treat symptoms that are now defined as AD in clinical treatment. However, the existing evidence for recommending DTD in clinical practice derives from studies that were methodologically flawed. In this study, we aim to determine the efficacy and safety of DTD in AD patients based on a rigidly randomized controlled trial. It will provide critical information on sample size and treatment regimen for conducting a full-scale clinical trial of DTD later.

Methods/Design: This study will be a double-blind, randomized, placebo-controlled, add-on trial. After a 2-week run-in period, eligible patients with mild to moderate AD will be recruited and given either DTD or placebo twice daily for 24 weeks with follow-up 6 weeks after the last treatment. An increase of four points or greater on the scores of Alzheimer's Disease Assessment Scale-cognitive subscale (ADAD-cog) will be considered as a positive primary outcome. Total scores of the ADAD-cog, the Chinese version of Mini-Mental State Examination (C-MMSE), and the Chinese version of the Disability Assessment for Dementia (C-DAD) score will be used as secondary outcomes. Adverse events will also be reported.
\end{abstract}

Discussion: This randomized trial will be the first rigorous empirical study on the efficacy of DTD for treating cognitive symptoms in AD patients. Its success will justify and warrant a large-scale clinical trial to further consolidate the evidence for DTD's efficacy in treating AD.

Trial registration: Chinese Clinical Trial Registry (ChiCTR-TRC-12004548, Date of registration: 22 November 2012)

Keywords: Alzheimer's disease, Randomized controlled trial, Di-Tan decoction, Chinese medicine

\section{Background}

Alzheimer's disease (AD) is an irreversible, progressive neurodegenerative disorder characterized by loss of cognitive functions, behavioral disturbances and daily living difficulties [1]. It is the most common type of dementia in the elderly [2]. Progressive atrophy is seen in all parts

\footnotetext{
* Correspondence: vctmok@cuhk.edu.hk; limin@hkbu.edu.hk

${ }^{2}$ Stroke and Clinical Neurosciences, Institute of Integrative Medicine, Department of Medicine and Therapeutics, Prince of Wales Hospital, The Chinese University of Hong Kong, Shatin, New Territories, Hong Kong ${ }^{1}$ School of Chinese Medicine, Hong Kong Baptist University, Kowloon Tong, Kowloon, Hong Kong

Full list of author information is available at the end of the article
}

of the brain, especially in the hippocampus region, due to nerve cell death and tissue loss [3]. It is estimated that the number of people suffering from dementia over the world will rise from 24.3 million in 2005 to 81.1 million in 2040 [4]. It is expected that a new case of AD will be diagnosed every 33 seconds in America by 2050 [5].

$\mathrm{AD}$ has a devastating impact not only on sufferers, but also on caregivers, their communities and the health care system in general [6]. While much research is being done on many aspects of $\mathrm{AD}$, including its basic biology, no drug has been found to alter the course of the disease [7]. Current pharmacological treatment for AD such as 
cholinesterase inhibitors (ChEIs) and memantine are symptomatic and may commonly associated with some side effects, such as nausea, vomiting, dizziness and anorexia [8]. Given that the efficacy is mild but with some side effects, patients often seek alternative treatments [9].

In traditional Chinese medicine (TCM), $\mathrm{AD}$ is described in terms of TCM principles; it could be caused by (1) a deficiency of vital energy of the kidney, heart and spleen or (2) the stagnation of blood and/or phlegm [10]. The guidelines that classified dementia into different subtypes according to the TCM theory was published in 1990, and symptoms of "phlegm turbidity obstructing the orifices" (PTOO) is considered to be one of the major contributing factors to $\mathrm{AD}$ [11]. Thus, the primary TCM strategy for treating $\mathrm{AD}$ is to "resolve phlegm in order to open the orifices" [12]. The classic Chinese medicine formula "Di-tan decoction" (DTD) was developed by a famous TCM doctor, Dong $\mathrm{Su}$, in 1449 with the specific function of "resolving phlegm to open the orifices" [13]. It has been and still is frequently used to treat symptoms that are now defined as $\mathrm{AD}$ in clinical treatment with TCM $[14,15]$. In recent years, modern studies have attempted to verify the clinical effects of DTD in biochemical terms. Laboratory studies have shown that the memory impairment of $\mathrm{AD}$ model mice was significantly reduced by DTD $[16,17]$. In the brain tissue in mice treated with DTD, acetylcholine (Ach) and acetylcholine transferase (ChAT) were significantly increased, while acetylcholine esterase (AchE) was decreased [18]. The results of another study indicate that DTD may inhibit the decline of the dopamine content in brain tissue of model mice as well [19].

In our previous systematic review, there was no clinical trial using DTD as the main intervention for treating $A D$. For the AD clinical trials that had involved DTD as an adjunct intervention, we found that most of them support the efficacy of DTD in treating AD. However, the evidence for recommending DTD in clinical practice derives from studies that were methodologically flawed. None of them was a randomized controlled trial. They not only lacked randomization, blinding, and the use of control groups, but also failed to define inclusion and exclusion criteria and the quality of the intervention medicine [20]. Because of the methodological problems in previous studies, evidence to suggest that DTD is effective in treating AD is not strong. There is a lack of conclusive evidence to recommend a clinical trial.

As there is no basic information of DTD to conduct a comprehensive $\mathrm{AD}$ clinical trial, a rigorous pilot study, which focuses on the efficacy and safety of DTD for treating $\mathrm{AD}$, will be needed before conducting a full-scale clinical trial. In this study, we will evaluate the efficacy and safety of DTD in patients with AD in a randomized controlled trial. This study will be a randomized, double- blinded, placebo-controlled study, with restrictive inclusion and exclusion criteria and a clearly defined quality control intervention medicine. We hope the results from this study will provide critical information on sample size and treatment regimen for conducting a full-scale clinical trial later.

\section{Methods/Design Study design}

This study will be a double-blinded, randomized, placebocontrolled, add-on trial. Patients with mild to moderate $\mathrm{AD}$ will be randomly assigned to receive 24 weeks of either active herbal treatment or placebo (in a 1:1 ratio); they will be followed for a further 6 weeks' observation period without treatment. This clinical study will be carried out at the Hong Kong Baptist University Chinese Medicine Specialty Centre. It has been approved by the Ethics Committee of the Hong Kong Baptist University's Institutional Review Board (code: HASC/11-12/24) and registered with the Chinese Clinical Trial Registry (ChiCTR-TRC-12004548). If there is any amendment to the protocol, approval must be again sought from the Ethics Committee. Written informed consent will be obtained from every patient before participation in any study-related activity. The protocol design is based on the guidelines of Consolidated Standards of Reporting Trials (CONSORT) and Standard Protocol Items: Recommendations for Interventional Trials (SPIRIT) [see Additional file 1], and study results will be reported according to these guidelines as well.

\section{Participants}

Inclusion criteria are as follows: Adults who (1) have been clinically diagnosed with $\mathrm{AD}$ based on the criteria of National Institute of Neurological and Communicative Disorders and Stroke and Alzheimer's Disease and Related Disorders Association (NINCDS/ADARA) [21], and (2) present symptoms classified as PTOO [see Additional file 2] as defined by the Guidance for Clinical Research of New Chinese Herbal Medicine [22] during a screening visit, will be eligible. The diagnostic criteria of PTOO include dementia, heavy-headedness and spitting phlegm. Additional inclusion criteria are as follows: (1) mild to moderate dementia with $\geq 2$ on Clinical Dementia Rating Scale (CDR) [23]; (2) receiving a stable dose of anti-dementia medication (that is, Donepezil, Rivastigmine and Memantine) for at least 4 weeks before the start of treatment; and (3) normal liver and renal function.

Exclusion criteria are as follows: Patients who also have any other type of dementia (that is, vascular dementia), and/or other neurodegenerative disorder (that is, Parkinson's disease), depression (defined by a score of $\geq 8$ on the 15 -item Chinese version of the Geriatric Depression Scale [24]), or who are unwilling to cooperate 
with treatment procedures will be excluded. For those who have participated in other trials within 30 days of the start of this trial as well as women who are pregnant or breastfeeding will also be excluded.

\section{Study medication}

The herbal medicine under study is DTD (Table 1). It is composed of Arisaema Cum Bile (DanNanXing in Chinese), Pinelliae Rhizoma (FaBanXia in Chinese), Aurantii Immaturus Fructus (ZhiShi in Chinese), Poria (FuLing in Chinese), Citri Reticulatae Pericarpium (ChenPi in Chinese), Acori Tatarinowii Rhizoma (ShiChangPu in Chinese), Ginseng Radix (RenShen in Chinese), Bambusae in Taeniam Caulis (ZhuRu in Chinese), Glycyrrhizae Radix (GanCao in Chinese), Zingiberis Recens Rhizoma (ShengJiang in Chinese) and dextin [13]. The test report of the DTD quality control is attached in the Additional file 3. The placebo is made of caramel (2\%), gardenia yellow pigment $(0.05 \%)$, sunset yellow $(0.02 \%)$, tartrazine $(0.02 \%)$, dextrin (95\%) and broadleaf holly leaf $(2.91 \%)$ [25]. The granules were produced in a single batch (DTD batch no.: A12074 Placebo batch no.: A120817) strictly in compliance with standards of Good Manufactory Practice (GMP) and Chinese Pharmacopoeia 2010 to ensure the stability and homogeneity of the composition as produced by PuraPharm Pharmaceuticals Company Limited. The chemical compositions of the final products were analyzed for contamination with heavy metals, toxic elements, microbe and pesticide residues; both final products were analyzed for stability and adherence to quality standards. The active treatment granules and the placebo granules have identical appearance and smell, and both have been packed in sealed opaque aluminum sachets and put in zip lock bags (10 sachets each). Only the treatment code is printed on the package to ensure successful blinding of patients [26]. One sachet contains $13.5 \mathrm{~g}$, a dosage equivalent to $67.5 \mathrm{~g}$ herbs. All herbal and placebo granules will

Table 1 "Di-tan decoction" (DTD) composition

\begin{tabular}{lll}
\hline Chinese name & Latin name & Percentage \\
\hline Dan Nan Xing & Arisaema Cum Bile & 11.42 \\
Ban Xia & Pinelliae Rhizoma & 11.42 \\
Zhi Shi & Aurantii Immaturus Fructus & 9.13 \\
Fu Ling & Poria & 9.13 \\
Chen Pi & Citri Reticulatae Pericarpium & 6.84 \\
Shi Chang Pu & Acori Tatarinowii Rhizoma & 4.55 \\
Ren Shen & Ginseng Radix & 4.55 \\
Zhu Ru & Bambusae in Taeniam Caulis & 3.19 \\
Gan Cao & Glycyrrhizae Radix & 2.29 \\
Sheng Jiang & Zingiberis Recens Rhizoma & 18.10 \\
Dextrin & - & 19.36 \\
\hline
\end{tabular}

be distributed by L.L. Chen with both written and verbal instructions for each participant. Patients will be instructed to take the granules orally by dissolving a sachet of granules in $150 \mathrm{ml}$ hot water, stirring well, then drinking the solution, two times per day, at least two hours apart from taking any routine Western medication. Patients will be allowed to discontinue the study granules temporarily if any adverse events occur. Patients will be instructed to report such events to a special e-mail account or by a direct telephone line. Additional treatments for a newly occurring illness, for example, flu or diarrhea, or adverse event during the study will be allowed but must be reported to the assessor.

\section{Recruitment procedures}

Three methods will be used to recruit participants with AD. The first source of candidates will be referral from two public Western medicine hospitals in Hong Kong (Prince of Wales Hospital and Queen Elizabeth Hospital) from our co-investigator (Co-I), V. Mok, and our research teammate, A.L.T. Chan. The second source will be referral from the Chinese medicine clinic of Hong Kong Baptist University. A third source of candidates will be those who respond to advertisements published in local newspapers and newsletters of local AD societies.

Figure 1 shows the schedule of enrollment. We plan to recruit at least 40 patients, which will allow 20 for the DTD group and 20 for the placebo group in this study. All patients diagnosed with $\mathrm{AD}$ will be referred to a Chinese medicine doctor who is the Principle Investigator of the study (M. Li), or to a Research Assistant (K.K. Chua), for further assessment and recruitment. The aim, procedures, nature of study and possible side effects of DTD will be explained by the PI or RA; then, each subject will be asked to sign a written consent [see Additional file 4] to take part in the study. Patients will be informed that they are free to withdraw at any time during the study.

All patients will undergo a 2-week run-in period, in which they have to keep their Western medicine and supplement dosages fixed. Their blood will be tested to check liver function (alanine transaminase (ALT)/serum glutamic pyruvic transaminase (SGPT), aspartate transaminase (AST)/ serum glutamic oxaloacetic transaminase (SGOT), alkaline phosphatase, gamma glutamyltransferase, total bilirubin, total protein, albumin) and renal function (urea, creatinine, sodium, potassium, chloride, bicarbonate) in a lab test center designated for the project.

\section{Randomization and masking}

The randomization sequence will be generated by SPSS 19.0 package (SPSS, Chicago, IL). The sequence will be password-protected and kept in a computer by L.L. Chen. Group allocation will be simple randomization in a ratio of 1:1 to either active treatment group or placebo 


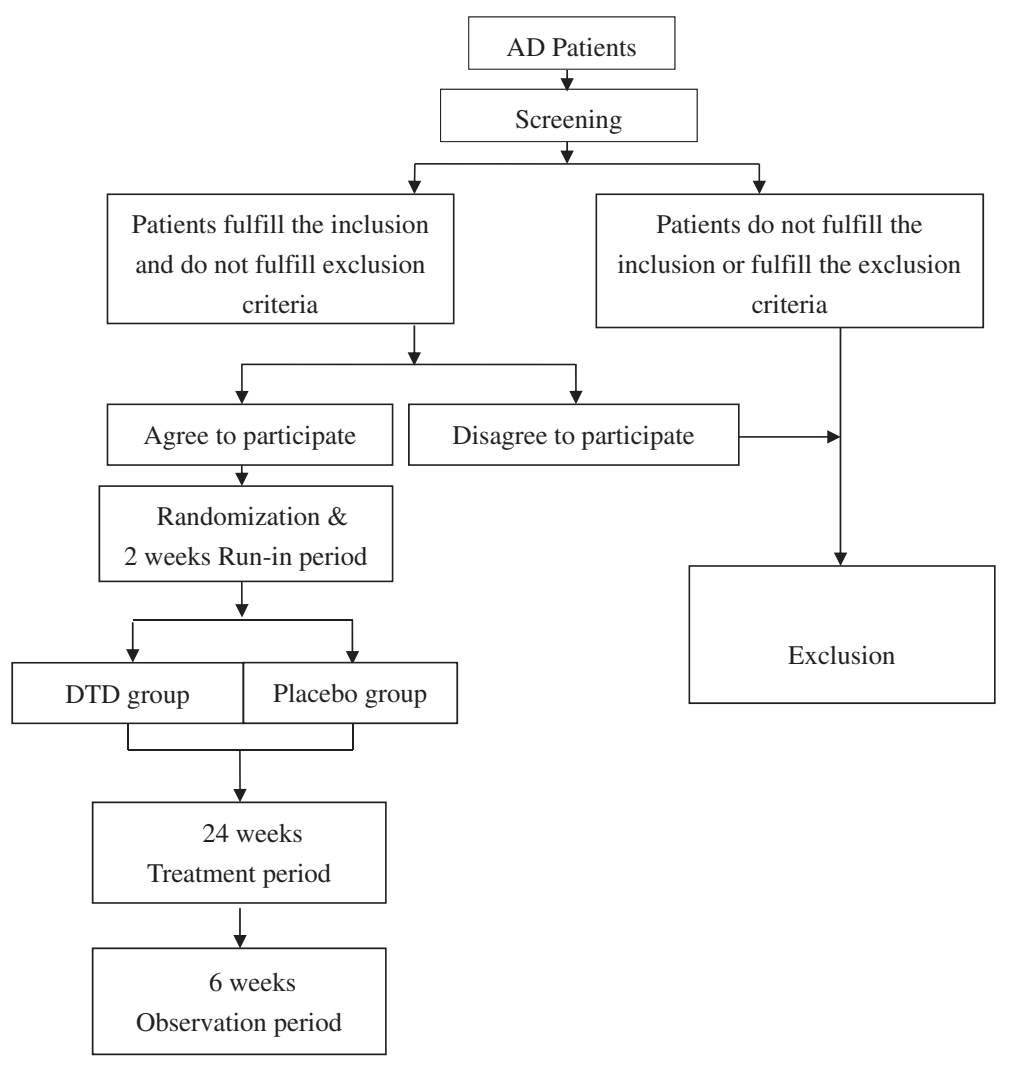

Figure 1 Schedule of enrollment.

group. The number sequences will be kept in sealed opaque envelopes and distributed to assessors. Patients, investigators and all sponsoring parties will be masked to treatment allocation until the end of the study. When there is a serious adverse event, the event will be discussed between the principal investigator Chinese medicine expert $\mathrm{M}$. $\mathrm{Li}$, and the co-investigator neurology specialist V. Mok to consider unblinding.

\section{Primary outcome and its assessment}

A positive primary outcome of this study will be an increase of at least four points in the Alzheimer's Disease Assessment Scale-cognitive subscale (ADAD-cog) [27] total score; this is considered to be a clinically meaningful increase [28]. Total scores of the ADAD-cog, Chinese version of Mini-Mental State Examination (C-MMSE) $[29,30]$ and Chinese version of Disability assessment for dementia (C-DAD) score [31] will be used as secondary outcomes.

Outcome measurements will be carried out during the study visits at weeks 0,12 (half of treatment), 24 (end of treatment) and 30 (end of observation period). Safety assessment, which includes reporting of adverse events (AEs), measurement of vital signs and physical examination, will be carried out throughout the study. In addition, laboratory safety screening of liver and renal function will be performed at week 24. Bilingual assessors, trained by the same neurology specialist V. Mok and clinical psychologist A. Wong, will be blind to the treatment allocation.

Clinical assessment will be rescheduled within 7 days after the scheduled clinical visit in case of any unexpected condition such as severe weather conditions. Phone call reminders will be given to the caregivers one day before the days of assessment.

A home diary will be given to each study participant's caregiver to record treatment and changes in the participant's medical condition. Formal instruction for the home diary will be given during the first visit. Revision and checking of the diary will be carried out with the caregiver during each formal visit by the assessors. Compliance in taking the treatments will be determined by the record of the diary and the number of the returned medicine/placebo packages.

\section{Data management and monitoring}

All the data will be entered and stored in a passwordprotected computer. Hardcopy will be kept in a locker. No interim analyses will be carried out. To ensure high quality of the data, a double data entry method will be used. All documents and collected data will be kept for 7 years after the finish of study and then will be 
destroyed. The protocol and statistical results will be published in a scientific journal where the public will have access to all the results.

A data monitoring committee (DMC) will be formed and will include at least two faculty members from the participating universities, who are independent of the research team and who will not participate in any other aspect of the study. The data management process will be monitored by DMC regularly. All the data will be frozen and then locked to prevent further editing after the validation by the DMC. Only the DMC, the study research assistant and the principal investigator will have access to the final dataset.

\section{Statistical analysis}

The proportion of patients with at least a four-point change in ADAS-cog and MMSE and the scores on CDAD will be compared between the active treatment and placebo groups using the Chi-Squared test and independent sample $t$ test, respectively. Missing data will be input using the last-observation-carried-forward (LOFC) approach. All patients randomized with at least one post-randomization measurement will be included in the primary analysis to follow the intention-to-treat principle. Analyses will be done with SPSS 19.0 package (SPSS, Chicago, IL).

To get a more comprehensive picture of the efficacy of DTD, a post-hoc analysis of the differences in the change of ADAS-cog and C-DAD sub-scores of the active treatment group will be compared between the active and placebo groups. To reduce the number of statistical comparisons, analyses will be performed with a hierarchical approach. To begin, the scores of ADAS-cog at week 24 (end of treatment) for the active treatment and placebo groups will be compared. If the difference is deemed statistically significant at a 2 -sided $\alpha$-level of 0.05 , the scores of ADAS-cog at week 12 (half of treatment) and week 30 (end of observation period, that is, without treatment) will be compared between groups. C-DAD will be analyzed in the same manner as ADAS-cog.

\section{Compliance strategy}

This will be a 30 -week clinical trial, in which subjects will need to take study medication for 24 weeks with 8 regular visits (week $0,4,8,12,16,20,24$ and 30). In order to maximize subjects' compliance, first, we will have a thorough consent process for all participants; we will explain in detail the study schedule, potential side effects of treatment, and the responsibilities of the subjects. Second, we will screen potential subjects carefully during a 2-week run-in period in order to exclude ineligible patients or patients who are unlikely to comply with treatment regimen before randomization. Third, we will try to prevent dropouts by providing ongoing support to patients. A special e-mail account and a direct telephone line set up for this clinical trial will enable the study team to personally communicate with the patients. An information sheet will be given to each participant providing them and their caregivers with means of urgent contact. Extra visits and free medical care will be arranged for any participant who feels harmed by the trial protocol. Fourth, this is an add-on design [32], which means that the study treatment is added to their existing treatment. Certainly those participants receiving the placebo should not experience any difference in their condition, while those receiving the DTD should improve or at least remain the same.

\section{Early termination}

The trial will be terminated for a specific participant in the event of any of the following: (1) participant develops severe adverse side effect(s); (2) participant shows hypersensitivity towards DTD; (3) participant develops some other life-threatening condition or disease; or (4) participant chooses to participate in another Chinese medicine research project. In addition, any participant may voluntarily withdraw.

The whole research plan will be terminated under the following circumstances: (1) presence of serious adverse effect(s) related to Chinese herbal medicine with supportive evidence or (2) completion of all follow-up assessments.

\section{Discussion}

$\mathrm{AD}$, as a progressive neurodegenerative disorder, has devastating impacts not only on patients but also on family members, friends, and caregivers. A cure would bring immeasurable relief and hope to countless thousands. Unfortunately, western pharmacological research has been unsuccessful in finding effective treatment without serious side effects [8]. In this case, Chinese medicine may have something to offer. Chinese medicine has been used to treat neurological diseases for thousands years with efficacy, safety, and relatively few side effects - albeit without rigorous and objective testing [33].

This randomized trial will be the first rigorous testing of DTD for the treatment of AD patients. Success in this clinical trial will provide evidence acceptable to the scientific community of the potential of DTD to become an effective medicine for AD. The success of this study would represent justification and impetus for a largescale clinical trial to further consolidate the evidence for the use of DTD in the treatment of AD.

It is noted that the sample size calculation does not apply to this study. Normally, 10 to 20 patients in each group would be sufficient to implement the method of a pilot study [34]. Since there is no previous data indicating the sample size needed to unequivocally determine the effect of DTD by the ADAS-cog, the evidence will 
still be weak even if we have a positive result. Nevertheless, the one aspect of this study that could undermine its value is sample size. There is no information on what sample size is needed to give statistically significant results. On the other hand, this study could find out what specific symptoms, like mood or cognitive, that DTD may improve. This information should be an important evidence for a much precise clinical trial with larger sample size in the future.

In conclusion, the results of this study are expected to provide evidence for the efficacy and safety of the Chinese herbal formula, DTD, for treatment of AD patients who also meet the diagnostic criteria of "phlegm obstructing the orifices" syndrome according to TCM diagnostics. It may also provide a new direction for the clinical research on $\mathrm{AD}$ and TCM.

\section{Trial status}

The trial has been approved and registered. At the time of manuscript submission, the study has been actively enrolling subjects, and so far has a total of 36 subjects. The study is still ongoing. Herbal and placement packets have been produced.

\section{Additional files}

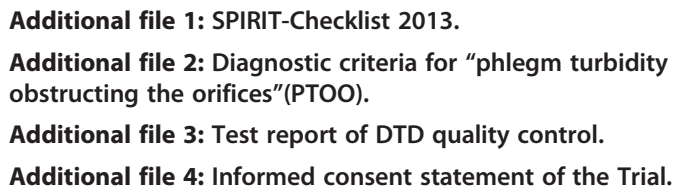

\section{Abbreviations}

Ach: acetylcholine; AchE: acetylcholine esterase; AD: Alzheimer's disease: ADAD-cog: Alzheimer's Disease Assessment Scale-cognitive subscale; AEs: adverse events; ALT: alanine transaminase; AST: aspartate transaminase; C-DAD: Chinese version of Disability Assessment for Dementia; CDR: Clinical Dementia Rating Scale; ChAT: acetylcholine transferase; ChEls: cholinesterase inhibitors; C-MMSE: Chinese version of Mini-Mental State Examination; CONSORT: Consolidated Standards of Reporting Trials; DMC: Data monitoring committee; DTD: Di-tan decoction; LOFC: last-observation-carried-forward; NINCDS/ADARA: National Institute of Neurological and Communicative Disorders and Stroke and Alzheimer's Disease and Related Disorders Association; PTOO: phlegm turbidity obstructing the orifices; SGOT: serum glutamic oxaloacetic transaminase; SGPT: serum glutamic pyruvic transaminase; SPIRIT: Standard Protocol Items: Recommendations for Interventional Trials; TCM: Traditional Chinese Medicine.
\end{abstract}

\section{Competing interests}

All authors confirm that they have no competing interests in this paper. Neither the funding agency nor any outside organization had any role in study design or manuscript preparation.

\section{Authors' contributions}

The authors' contributions are as follows: ML conceived the project and will coordinate all efforts in the clinical trial; VM will monitor the trial; AW and PWLK contributed to the design of the trial; KKC and PWLK will help carry out and monitor the clinical trial; VM and ALTC referred and recruited patients; LLC will distribute the medicine; and KKC, JXS, JHL, VM and ML wrote this paper. All authors read and approved the final version of this manuscript.

\section{Acknowledgements}

This work is supported by an anonymous donation clinical research grant (GDS 0216), PuraPharm International (H.K.) Ltd. and Dr. Ho Tzu-leung's Foundation. This study was also support by HMRF 12132091 from the Food and Health Bureau, Hong Kong Government, research grants (IRMS/12-13/1A, MPCF008-2014/2015) and Mr. \& Mrs. Ko Chi Ming Centre for Parkinson's Disease Research from Hong Kong Baptist University. The authors would like to thank Dr. Feng Sun, Ms. Vicky Keng from Clinical Division, School of Chinese Medicine, Hong Kong Baptist University and Hong Kong Alzheimer's Disease Association for their great support on this clinical study. The authors would also like to thank Dr. Martha Dahlen for her English editing of this manuscript.

\section{Author details}

${ }^{1}$ School of Chinese Medicine, Hong Kong Baptist University, Kowloon Tong, Kowloon, Hong Kong. ${ }^{2}$ Stroke and Clinical Neurosciences, Institute of Integrative Medicine, Department of Medicine and Therapeutics, Prince of Wales Hospital, The Chinese University of Hong Kong, Shatin, New Territories, Hong Kong. ${ }^{3}$ Divisions of Neurology \& Geriatrics, Department of Medicine, Queen Elizabeth Hospital, Kowloon, Hong Kong. ${ }^{4}$ State Key Laboratory of Quality Research in Chinese Medicine, Institute of Chinese Medical Sciences, University of Macau, Taipa, Macau.

Received: 20 November 2014 Accepted: 9 April 2015

Published online: 30 April 2015

\section{References}

1. Mattson MP. Pathways towards and away from Alzheimer's disease. Nature. 2004:430:631-9.

2. Querfurth HW, LaFerla FM. Alzheimer's Disease. New Engl J Med. 2010;362:329-44.

3. Gu XM, Jiang ZF, Huang HC. Magnetic resonance imaging of Alzheimer's disease: from diagnosis to therapeutic evaluation. Chin J Integr Med. 2010;16:276-82.

4. Ferri $C P$, Prince $M$, Brayne C, Brodaty H, Fratiglioni L, Ganguli M, et al. Global prevalence of dementia: a Delphi consensus study. Lancet. 2005;366:2112-7.

5. Thies W, Bleiler L. 2013 Alzheimer's disease facts and figures. Alzheimers Dement. 2013;9:208-45.

6. Hampel H, Prvulovic D, Teipel S, Jessen F, Luckhaus C, Frolich L, et al. The future of Alzheimer's disease: the next 10 years. Prog Neurobiol. 2011:95:718-28.

7. Carrillo MC, Brashear HR, Logovinsky V, Ryan JM, Feldman HH, Siemers ER, et al. Can we prevent Alzheimer's disease? Secondary "prevention" trials in Alzheimer's disease. Alzheimers Dement. 2013;9:123-131.e121.

8. Massoud F, Leger GC. Pharmacological treatment of Alzheimer disease. Can J Psych Rev. 2011;56:579-88.

9. Kelley BJ, Knopman DS. Alternative medicine and Alzheimer disease. Neurologist. 2008;14:299-306.

10. Ho YS, So KF, Chang RC. Drug discovery from Chinese medicine against neurodegeneration in Alzheimer's and vascular dementia. Chin Med. 2011;6:15.

11. Geriatric Society of the Traditional Chinese Medicine Society IMSotTCMS Diagnosis and rating standard of geriatric dementia. Chin J Rural Med Pharm. 1995;2:19-20.

12. Hui ML, Chua KK, Li M. A literature review on the treatment of Chinese medicine in neurodegenerative diseases through supplying kidney Qi, promote blood circulation and dispel phlegm. Hong Kong Chin Med J. 2011;6:35-40.

13. Li F. Chinese Medicine Formulae. Beijing: People's Medical Publishing House; 2002.

14. Yuan WLHJ, Luo CM. Review of Alzheimer disease treated by Chinese medicine. Mod J Integr Tradit Chin West Med. 2000;9:203-5.

15. Yu SMLL, Wang P. The theory of dementia treated by Ditan decoction. Fujian J TCM. 2007;38:49-50.

16. Liu LYR, Yan C, Dong H. Effects of modified Ditan decoction on the neural degeneration and neuroethology in model mice with learning defect. Henan Tradit Chin Med. 2010;30:553-4.

17. Liu LLY, Jiang YM, Yu SM. Experimental study on effect of Ditan decoction on ethology in model rats with Alzheimer's disease. Mod J Integr Tradit Chin West Med. 2010;19:3154-8. 
18. Liu LYY, Yu SM, Li Y. Effect of Ditan decoction on central cholinergic system in model rats with Alzheimer's disease. Hubei J TCM. 2009;31:10-1.

19. Liu LDH, Jiang YM, Yu SM. Effects of Ditan decoction on learning and memory function in multiple models. Chin I Guang Ming Chin Med. 2010;25:1364-7.

20. Chua KK, Chen LL, Liu LF, Kumar DS, Lu JH, Li M. Efficacy of classic Chinese medicine formula Ditan Decoction () for Alzheimer's disease. Chin J Integr Med. 2014; DOI: 10.1007/s11655-014-1346-X.

21. McKhann G, Drachman D, Folstein M, Katzman R, Price D, Stadlan EM. Clinical diagnosis of Alzheimer's disease: report of the NINCDS-ADRDA Work Group under the auspices of Department of Health and Human Services Task Force on Alzheimer's Disease. Neurology. 1984;34:939-44.

22. Zheng XY. The Guidance for Clinical Research of New Chinese Herbal Medicine. Beijing: Chinese Medical Scientific Publishers; 2002.

23. Hughes $C P$, Berg L, Danziger WL, Coben LA, Martin RL. A new clinical scale for the staging of dementia. Br J Psychiatry. 1982;140:566-72.

24. Wong MT, Ho TP, Ho MY, Yu CS, Wong YH, Lee SY. Development and inter-rater reliability of a standardized verbal instruction manual for the Chinese Geriatric Depression Scale-short form. Int J Geriatr Psychiatry. 2002;17:459-63.

25. Tang XD, Bian LQ, Gao R, Guan SJ. Exploration into the preparation of placebos used in Chinese medicinal clinical trial. Chin J Integr Tradit West Med. 2009;29:656-8

26. Zhong LL, Cheng CW, Chan Y, Chan KH, Lam TW, Chen XR, et al. Chinese herbal medicine (Ma Zi Ren Wan) for functional constipation: study protocol for a prospective, double-blinded, double-dummy, randomized controlled trial. Trials. 2013;14:366

27. Graham DP, Cully JA, Snow AL, Massman P, Doody R. The Alzheimer's Disease Assessment Scale-Cognitive subscale: normative data for older adult controls. Alzheimer Dis Assoc Disord. 2004;18:236-40.

28. Stern RG, Mohs RC, Davidson M, Schmeidler J, Silverman J, Kramer-Ginsberg E, et al. A longitudinal study of Alzheimer's disease: measurement, rate, and predictors of cognitive deterioration. Am J Psychiatry. 1994;151:390-6.

29. Chiu HFLH, Chung WS, Kwong PK. Reliability and validity of the Cantonese version of the Mini-Mental State Examination - a preliminary study. J Hong Kong Coll Psychiatrists. 1994;2:25-8.

30. Soto ME, Andrieu S, Cantet C, Reynish E, Ousset PJ, Arbus C, et al. Predictive value of rapid decline in mini mental state examination in clinical practice for prognosis in Alzheimer's disease. Dement Geriatr Cogn Disord. 2008;26:109-16.

31. Mok CC, Siu AM, Chan WC, Yeung KM, Pan PC, Li SW. Functional disabilities profile of Chinese elderly people with Alzheimer's disease - a validation study on the Chinese version of the disability assessment for dementia. Dement Geriatr Cogn Disord. 2005;20:112-9.

32. Little RJ, D'Agostino R, Cohen ML, Dickersin K, Emerson SS, Farrar JT, et al. The prevention and treatment of missing data in clinical trials. N Engl J Med. 2012;367:1355-60.

33. Man SC, Durairajan SS, Kum WF, Lu JH, Huang JD, Cheng CF, et al. Systematic review on the efficacy and safety of herbal medicines for Alzheimer's disease. J Alzheimers Dis. 2008;14:209-23.

34. Hertzog MA. Considerations in determining sample size for pilot studies. Res Nurs Health. 2008;31:180-91.

\section{Submit your next manuscript to BioMed Central and take full advantage of:}

- Convenient online submission

- Thorough peer review

- No space constraints or color figure charges

- Immediate publication on acceptance

- Inclusion in PubMed, CAS, Scopus and Google Scholar

- Research which is freely available for redistribution 